\title{
Inhibitory Effects of Chrysanthemum (Chrysanthemum morifolium Ramat.) Extract and Its Active Compound Isochlorogenic Acid A on Sarcopenia
}

\author{
Dowan Kwon ${ }^{1}$, Changhee Kim 1 , Yu Kyong Woo², and Jae-Kwan Hwang ${ }^{1,2}$ \\ ${ }^{1}$ Department of Biotechnology, College of Life Science and Biotechnology and ${ }^{2}$ Graduate Program in Bioindustrial Engineering, \\ Yonsei University, Seoul 03722, Korea
}

\begin{abstract}
Sarcopenia, age-related muscle atrophy, weakening muscle strength, and exercise capacity, generally accompany imbalances in protein metabolism. Chrysanthemum morifolium Ramat. extract (CME) and its active compound, isochlorogenic acid A (ICA), have been reported to have anti-oxidative, anti-diabetic, and neuroprotective effects. However, the roles of CME and IcA in the regulation of muscle protein turnover-related signaling pathways to attenuate sarcopenia have not been explored. In this study, we investigated CME and IcA based regulation of protein turnover in synthesizing muscle in vitro and in vivo. At the molecular level, CME and IcA promoted phosphorylation of PI3K/Akt and mTOR pathways, which stimulate synthesis of muscle proteins, and suppressed FoxO3a and E3 ubiquitin ligases during protein degradation. In vivo, CME and IcA increased grip strength, exercise capacity, muscle mass and volume, and cross-sectional area of myofibers in middle-aged C57BL/6J mice. These results suggest that CME and IcA may have roles as functional food supplements for delaying sarcopenia by enhancing muscle mass recovery and function.
\end{abstract}

Keywords: Chrysanthemum morifolium, isochlorogenic acid A, protein turnover, sarcopenia

\section{INTRODUCTION}

Skeletal muscle accounts for $40 \% \sim 50 \%$ of total human body weight, and affects exercise performance, energy expenditure, and supporting other organs (Frontera and Ochala, 2015). The age-related decline in skeletal muscle mass is referred to as sarcopenia, which is characterized by a decrease in muscle strength and exercise capacity. These losses in muscle mass and function increase the likelihood of metabolic diseases, such as type II diabetes, obesity, and cardiovascular diseases (Wolfe, 2006; Misra et al., 2010). Sarcopenia is accompanied by an imbalance of protein turnover and mitochondrial dysfunction, and regulating these factors is considered important for attenuating sarcopenia (Joseph et al., 2012).

Skeletal muscle mass is regulated by protein anabolism and catabolism. Phosphatidylinositol 3-kinase (PI3K) is crucial for Akt phosphorylation, which activates the mammalian target of rapamycin (mTOR) pathway. When mTOR is phosphorylated by PI3K/Akt pathway activation, expression of phosphorylated $70 \mathrm{kDa}$ ribosomal protein kinase S6 (p70S6K) and eukaryotic initiation factor 4 binding protein-1 (4EBP1) are upregulated, leading to skeletal muscle hypertrophy (Ge and Chen, 2012). The mechanism of protein degradation is mainly regulated by Forkhead box class $\mathrm{O}$ (FoxO), which is a transcription factor that regulates protein degradation by the ubiquitin-proteasome system (Schiaffino et al., 2013). FoxO3a influences muscle atrophy by regulating F-box (also called atrogin-1) and muscle RING-finger protein-1 (MuRF-1), which are two major E3 ubiquitin ligases that lead to ubiquitination of muscle proteins (Lagirand-Cantaloube et al., 2009; Sanchez et al., 2014).

Chrysanthemum morifolium Ramat., commonly called chrysanthemum, belongs to the Asteraceae family and has been studied for its anti-oxidative, anti-diabetic, and neuroprotective activities, and for its role in improving fatty liver disease (Cui et al., 2014; Kim, 2014; Yamamoto et al., 2015; Abushouk et al., 2017). C. morifolium contains caffeic acid, chlorogenic acid, and several types of hydroxycinnamic acid. Of the hydroxycinnamic acids in the flower, isochlorogenic acid A (IcA) is present in the highest concentration hence conferring biological activities (Kim et al., 2005; Chen et al., 2015; Hong et al., 2015). 
Although C. morifolium Ramat. extract (CME) and IcA are pharmacologically active, their effects on muscle synthesis have not been examined. In this study, we investigated whether standardized CME and IcA have inhibitory effects on sarcopenia.

\section{MATERIALS AND METHODS}

\section{Preparation of standardized CME and IcA}

Dried flowers of C. morifolium were supplied by Nutribiotech (Seoul, Korea). A specimen voucher was deposited at the Department of Biotechnology, Yonsei University (Seoul, Korea). Flowers were ground and extracted with $30 \%$ ethanol at $70^{\circ} \mathrm{C}$ for $4 \mathrm{~h}$ using a mechanical stirrer (MS3040; Misung Co., Seoul, Korea). CME was obtained by filtration and was concentrated using a rotary evaporator (Heidolph, Schwabach, Germany), giving a final yield of $23 \%(w / w)$. For standardization of CME, IcA was identified and quantified using a YL9100 HPLC system (Younglin Instrument Co., Ltd., Anyang, Korea) equipped with a Sunfire C18 column $(150 \mathrm{~mm} \times 4.6 \mathrm{~mm}$, $5 \mu \mathrm{m}$ i.d.; Waters Corp., Milford, MA, USA). Samples were injected at a volume of $20 \mu \mathrm{L}$ in gradient mobile phases composed of $0.1 \%$ phosphoric acid (phase A) and acetonitrile (phase B), 15\% 40\% B for 0 25 min, and then $40 \%$ B for $25 \sim 30 \mathrm{~min}$. The analysis was conducted at a flow rate of $0.5 \mathrm{~mL} / \mathrm{min}$ and UV detection at a wavelength of $330 \mathrm{~nm}$ at $25^{\circ} \mathrm{C}$. Chromatographic peaks of CME were identified by comparing with the retention time of pure IcA (Toronto Research Chemicals Inc., Toronto, ON, Canada). The standardized CME contained $2.17 \%(\mathrm{w} / \mathrm{w})$ of IcA, which is a bioactive compound.

\section{Cell culture}

L6 rat myoblasts were obtained from the American Type Culture Collection (Manassas, VA, USA). Cells were grown in Dulbecco's modified Eagle's medium (HyClone, Logan, UT, USA) supplemented with $10 \%$ fetal bovine serum (Gibco, Gaithersburg, MD, USA) and 1\% antibiotics $(100 \mathrm{U} / \mathrm{mL}$ penicillin A and $100 \mathrm{mg} / \mathrm{mL}$ streptomycin; Gibco) at $37^{\circ} \mathrm{C}$ in an atmosphere of $5 \% \mathrm{CO}_{2}$. After cells reached approximately $80 \%$ confluence in plates, the growth medium was changed to a differentiation medium containing $2 \%$ horse serum (HyClone) and $1 \%$ antibiotics. During the 6 days of differentiation, the medium was changed every 2 days. When cells were fully differentiated, cells were treated with $50 \mathrm{ng} / \mathrm{mL}$ tumor necrosis factor- $\alpha$ (TNF- $\alpha$; PeproTech, Rocky Hill, NJ, USA), CME ( 1 and $10 \mu \mathrm{g} / \mathrm{mL}$; denoted as CME 1 and 10 , respectively) and IcA ( 1 and $10 \mu \mathrm{M}$; denoted IcA 1 and 10, respectively) overnight.

\section{Cell viability}

L6 myoblasts were seeded onto a 24-well plate, and their viability was determined using 3-(4,5-dimethylthiazol-2yl)-2,5-diphenyltetrazolium bromide (MTT) colorimetric assays. After treatment with CME and IcA for $24 \mathrm{~h}$, L6 myoblasts were incubated with MTT (Sigma-Aldrich Co., St. Louis, MO, USA) for $4 \mathrm{~h}$ and washed with Dulbecco's phosphate-buffered saline. After drying the solvent from the wells, 99\% dimethyl sulfoxide (Samchun Chemical Co., Seoul, Korea) was added to dissolve insoluble formazan, and the absorbance was measured at $540 \mathrm{~nm}$ using a VersaMax ${ }^{\mathrm{TM}}$ tunable microplate reader (Molecular Devices Inc., Sunnyvale, CA, USA).

\section{Animal experiments}

Six-week-old C57BL/6J mice (Dae Han BioLink Co., Ltd., Eumseong, Korea) and 13-month and 14-month-old mice (Korea Basic Science Institute, Gwangju, Korea) were housed at $25^{\circ} \mathrm{C}, 50 \% \sim 60 \%$ humidity, and 12 -h day/night cycle conditions. Six-week-old C57BL/6J mice (young group) were used as negative controls. Thirteen-month and 14-month-old C57BL/6J mice were randomly divided into four groups: (i) middle-aged (MA); (ii) CME 50 (50 mg/kg/d); (iii) CME 200 (200 mg/kg/d); and (iv) IcA $20(20 \mathrm{mg} / \mathrm{kg} / \mathrm{d})$. After circulation for 1 week, CME and IcA were orally administered daily for 8 weeks. The mice in the young and MA groups were orally administered saline. After 8 weeks, mice were euthanized by cardiac puncture under anesthesia and the gastrocnemius (GA), soleus (SOL), tibialis anterior (TA) and extensor digitorum longus (EDL) muscles were isolated from the hindlimbs and stored at $-70^{\circ} \mathrm{C}$. This animal experiment was approved by the Institutional Animal Care and Use Committee (IACUC) of Yonsei University (permit number: IACUC-A-201803-203-02).

\section{Treadmill tests}

Running time and running distance were measured using a treadmill (Panlab, Barcelona, Spain). For acclimation, mice were made to run at a speed of $20 \mathrm{~cm} / \mathrm{s}$ at $0^{\circ}$ inclination for $20 \mathrm{~min}$. In the actual tests, the mice were made to run at a speed of $20 \mathrm{~cm} / \mathrm{s}$ at $0^{\circ}$ inclination, and the speed was increased to $45 \mathrm{~cm} / \mathrm{s}$ at the rate of $1 \mathrm{~cm} / \mathrm{s}$ per min, which was maintained until the mice were exhausted. A 0.2-mA electric shock was delivered to mice, which did not physically injure the animals. Tests were terminated when mice were unable to run with a continuous electric shock for $10 \mathrm{~s}$.

\section{Grip strength}

Grip strength tests were performed using a grip strength meter (Bioseb, Vitrolles, France) equipped with a grip bar. Each mouse was held on a grid by grabbing its tail until it released the grip bar. Forelimb and fore/hindlimb 
grip strength were measured at least five times to calculate the average value.

\section{Micro-computed tomography imaging}

To quantify muscle volume, micro-computed tomography (micro-CT) images were captured using an animal positron emission tomography/CT/single-photon emission tomography system (Siemens, Washington, DC, USA) at Pohang Technopark (Pohang, Korea). The images were analyzed using the Inveon Research Workplace software (Siemens, Healthineers, Erlangen, Germany).

\section{Histological analysis}

Hematoxylin and eosin (H\&E) staining was used to measure the myofiber cross-sectional area (CSA) of the GA muscles, which were fixed with $10 \%$ formalin buffer (Junsei Chemical Co., Ltd., Tokyo, Japan). The GA muscles were cut and embedded in paraffin to produce paraffin blocks. Each paraffin block was mounted on a slide, and the paraffin sections were stained with H\&E. The stained muscle fiber images were observed, captured and analyzed using an inverted microscope (CK40; Olympus, Tokyo, Japan) equipped with an eXcope T500 camera (magnification, $\times 200$; DIXI Science, Daejeon, Korea). The myofiber CSA was quantified using Image J software (National Institutes of Health, Bethesda, MD, USA).

\section{Western blot assays}

Protein extracts were collected by lysing L6 myotubes and GA tissues with lysis buffer (NP40; Elpis Biotech Inc., Daejeon, Korea) supplemented with a protease inhibitor cocktail (Sigma-Aldrich). Bradford assays were used to determine lysate protein concentrations. After protein quantification, equal amounts of proteins were separated by sodium dodecyl sulfate-polyacrylamide gel electrophoresis on $10 \%$ gels and transferred to nitrocellulose membranes (Whatman, Dassel, Germany). The membranes were blocked with 5\% skim milk (Becton Dickinson, Franklin Lakes, NJ, USA) in Tris-buffered saline with Tween-20 at room temperature for $30 \mathrm{~min}$. Next, membranes were incubated overnight at $4^{\circ} \mathrm{C}$ with primary antibodies against phosphorylated PI3K, Akt, mTOR, p70S6K, 4EBP1, FoxO3a (p-PI3K, p-Akt, p-mTOR, p-p70S6K, p-4EBP1, and p-FoxO3a, respectively), PI3K, Akt, mTOR, p70S6K, 4EBP1, FoxO3a and $\alpha$-tubulin (1:1,000 dilution; Cell Signaling, Beverly, MA, USA). Bound primary antibodies were detected by incubating with horseradish peroxidase-linked secondary antibodies (1:5,000 dilution; Bethyl Laboratories Inc., Montgomery, TX, USA) at $4^{\circ} \mathrm{C}$ for $2 \mathrm{~h}$. Antibody signals were detected using an enhanced chemiluminescence detection system (Amersham Biosciences, Little Chalfont, UK) and visualized using the G:BOX image analysis system (Syngene, Cambridge, UK). Results were quantified using Image J soft- ware (National Institutes of Health). The levels of phosphorylated proteins were normalized against the total levels of target proteins.

Reverse transcription-polymerase chain reaction (RT-PCR) mRNA was isolated from differentiated L6 cells and GA tissues using RNAiso Plus reagent (Takara, Kusatsu, Japan). Total mRNA was quantified spectrophotometrically using a Nanodrop instrument (Thermo Fisher Scientific Inc., Waltham, MA, USA) and cDNA was synthesized using Reverse Transcriptase Premix (Elpis Biotech Inc.). The cDNA was amplified using the Gene Amp PCR System 2700 (Applied Biosystems, Foster City, CA, USA) with HiPi PCR PreMix (Elpis Biotech Inc.) and primers using the following conditions: denaturation at $94^{\circ} \mathrm{C}$ for $30 \mathrm{~s}$, annealing for $1 \mathrm{~min}$, extension at $72^{\circ} \mathrm{C}$ for $1 \mathrm{~min}$, and final extension at $72^{\circ} \mathrm{C}$ for $5 \mathrm{~min}$. The primers used are listed in Table 1. The PCR products, stained with loading star (DyneBio, Seongnam, Korea), were separated by $1.5 \%$ agarose gel electrophoresis and detected by the G:BOX image analysis system (Syngene). The PCR products were quantified with the Image J software (National Institutes of Health).

\section{Statistical analysis}

Data are presented as the mean \pm standard deviation. Group differences were assessed using one-way analysis of variance (ANOVA), followed by Scheffe test, conducted with SPSS (version 23.0; IBM Corp., Armonk, NY, USA). Statistical significance was set at $P<0.05$.

\section{RESULTS}

\section{Effects of CME and IcA on cell viability}

The effects of CME and IcA on cell viability were evaluated using MTT assays. CME at $1 \mu \mathrm{g} / \mathrm{mL}$ and $10 \mu \mathrm{g} / \mathrm{mL}$ did not show any cytotoxic effects on L6 cells. IcA also

Table 1. Primer sequences used in reverse transcription-polymerase chain reaction analysis

\begin{tabular}{|c|c|c|c|}
\hline Origin & Gene & Direction & Sequence $\left(5^{\prime} \rightarrow 3^{\prime}\right)$ \\
\hline \multirow[t]{6}{*}{ Rat } & Atrogin-1 & Forward & CCCTGAGTGGCATCGCCCAA \\
\hline & & Reverse & AGGTCCCGCCCATCGCTCA \\
\hline & MuRF1 & Forward & CCAACGACATCTTCCAGGCT \\
\hline & & Reverse & TGGTGGCTGTTTTCCTTGGT \\
\hline & $\beta$-Actin & Forward & CTGTGTGGATTGGTGGCTCTAT \\
\hline & & Reverse & GTGTAAAACGCAGCTCAGTAACA \\
\hline \multirow[t]{6}{*}{ Mouse } & Atrogin-1 & Forward & CAGTGAGCCCTGCCATAACA \\
\hline & & Reverse & TCCCAAGTATGGAGCAGGGA \\
\hline & MuRF1 & Forward & GTCTGGAGGTCGTTTCCGTT \\
\hline & & Reverse & AGCAAGTAGGCACCTCACAC \\
\hline & $\beta$-Actin & Forward & TTACCAACTGGGACGACATG \\
\hline & & Reverse & ATACAGGGACAGCACAGCCT \\
\hline
\end{tabular}


did not affect cell viability at $1 \mu \mathrm{M}$ or $10 \mu \mathrm{M}$ (data not shown). Thus, further in vitro experiments were performed with $1 \mu \mathrm{g} / \mathrm{mL}$ and $10 \mu \mathrm{g} / \mathrm{mL} \mathrm{CME}$ and $1 \mu \mathrm{M}$ and $10 \mu \mathrm{M}$ IcA.
A
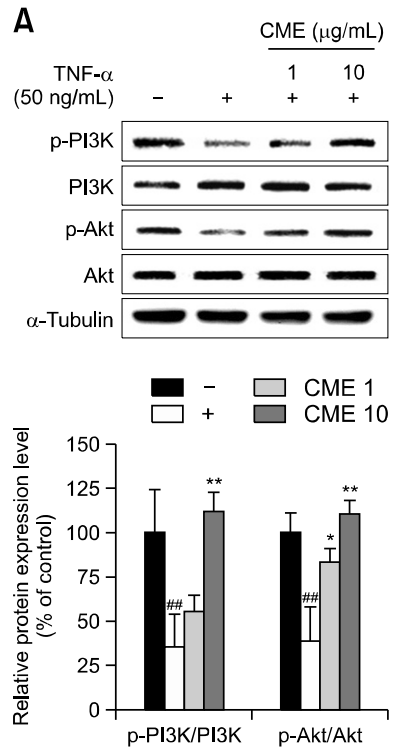

B
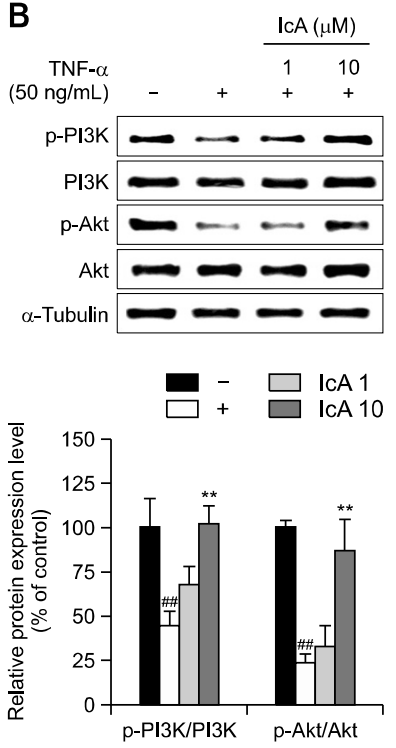

Effects of CME and ICA on protein synthesis in vitro

The effects of CME and IcA on regulation of muscle synthetic signaling pathways were investigated. L6 myotubes were treated with TNF- $\alpha$ to reduce phosphorylation of PI3K and Akt, which are major regulators of muscle protein synthesis. Western blot analysis showed that TNF- $\alpha$
C
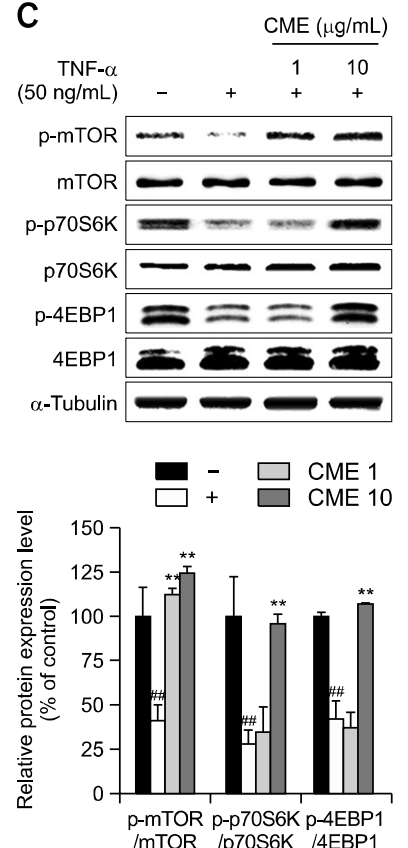

D
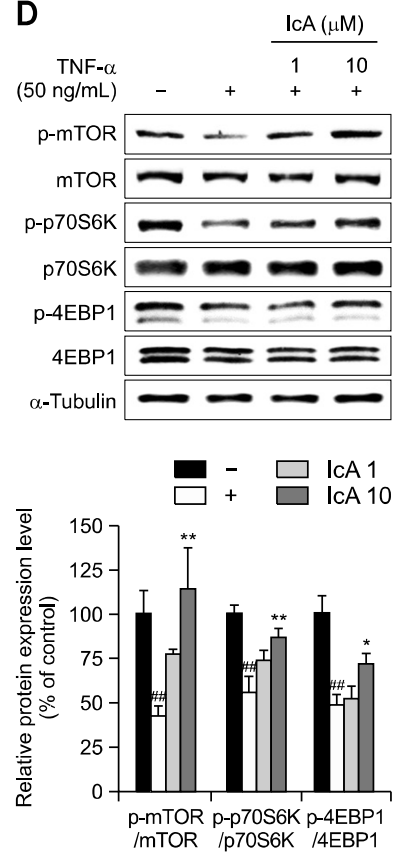

Fig. 1. Effects of Chrysanthemum morifolium Ramat. extract (CME) and isochlorogenic acid A (IcA) on muscle protein synthesis in L6 myotubes. L6 myotubes were treated with tumor necrosis factor- $\alpha$ (TNF- $\alpha)(50 \mathrm{ng} / \mathrm{mL}), \mathrm{CME}(1 \mathrm{and} 10 \mu \mathrm{g} / \mathrm{mL})$, and IcA $(1$ and $10 \mu \mathrm{M})$. (A and B) Relative protein levels of p-PI3K, PI3K, p-Akt, and Akt were measured using Western blot, with $\alpha$-tubulin as the housekeeping gene. ( $C$ and D) Relative protein expression of p-mTOR, mTOR, p-p70S6K, p70S6K, p-4EBP1, and 4EBP1 were measured using Western blot, with $\alpha$-tubulin as the housekeeping gene. Group differences were assessed using Scheffe multiple range test. ${ }^{\# \#} P<0.01$ (negative control group vs. TNF- $\alpha$-treated group) and ${ }^{*} P<0.05$ and ${ }^{* *} P<0.01$ (TNF- $\alpha$-treated group vs. CMEand IcA-treated groups).

A

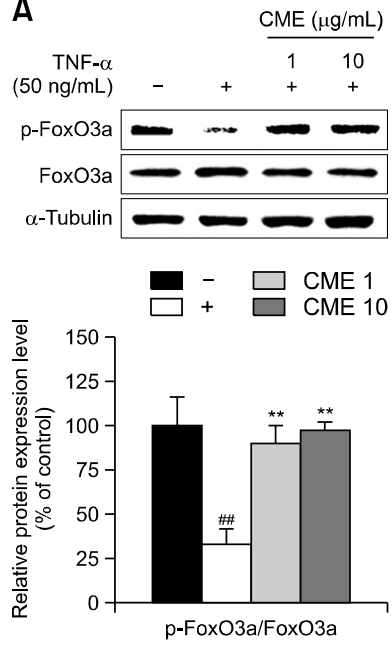

B
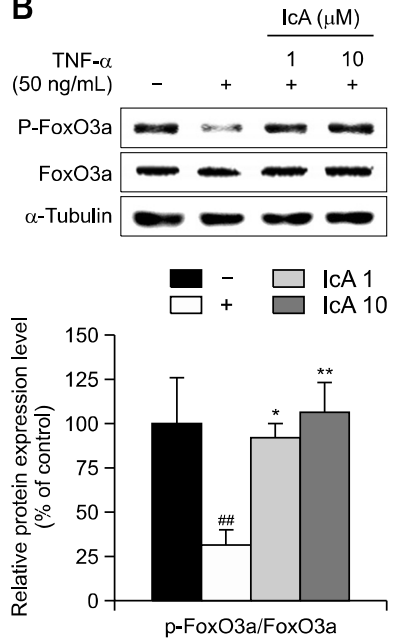

C

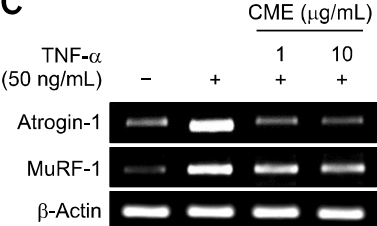

$\square+\square \operatorname{CME} 1$

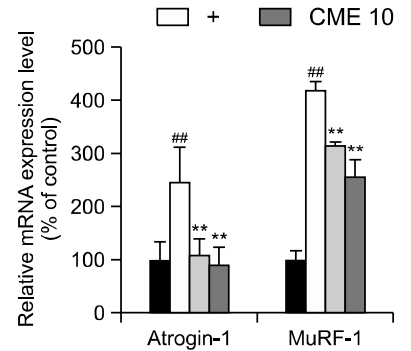

D

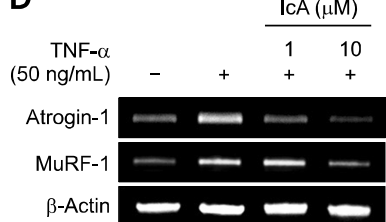

$\square+\square$ IcA 1

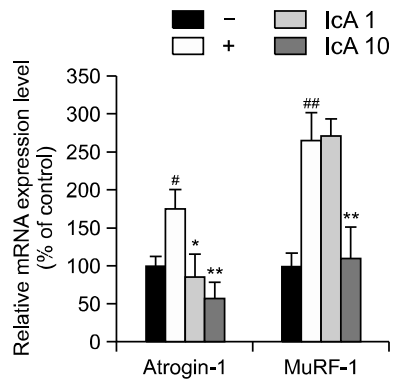

Fig. 2. Effects of Chrysanthemum morifolium Ramat. extract (CME) and isochlorogenic acid A (ICA) on muscle protein degradation in L6 myotubes. L6 myotubes were treated with tumor necrosis factor $-\alpha$ (TNF- $\alpha)(50 \mathrm{ng} / \mathrm{mL}), \mathrm{CME}(1 \mathrm{and} 10 \mu \mathrm{g} / \mathrm{mL})$, and IcA $(1$ and $10 \mu \mathrm{M})$. (A and B) Relative protein levels of $\mathrm{p}$-FoxO3a and FoxO3a were measured using western blot, with $\alpha$-tubulin as the housekeeping gene. ( $C$ and D) Relative mRNA levels of atrogin-1 and MuRF-1 were measured using reverse transcriptionpolymerase chain reaction, with $\beta$-actin as the housekeeping gene. Group differences were assessed using Scheffe multiple range test. ${ }^{\#} P<0.05$ and ${ }^{\# \#} P<0.01$ (negative control group vs. TNF- $\alpha$-treated group) and ${ }^{*} P<0.05$ and ${ }^{* *} P<0.01$ (TNF- $\alpha$-treated group vs. CME- and IcA-treated groups). 
reduced phosphorylation of $\mathrm{PI} 3 \mathrm{~K}$ and Akt, which are downstream targets of the PI3K/Akt pathway, including mTOR, p70S6K and 4EBP1 (Fig. 1). In the high concentration treatment groups, phosphorylation rates significantly increased compared with the TNF- $\alpha$ only treated groups, and recovered to similar levels as the TNF- $\alpha$ untreated cells.

\section{Effects of CME and IcA on protein degradation in vitro}

Expression associated with protein degradation were evaluated using western blot analysis. Phosphorylation of FoxO3a in TNF- $\alpha$-treated cells was inhibited by $67 \%$ relative compared with untreated cells. In CME 1 and IcA 1 treated cells, phosphorylated FoxO3a increased to $168 \%$ and $189 \%$, respectively, compared to TNF- $\alpha$-treated cells (Fig. 2A and 2B). In contrast, expression of atrogin-1 and MuRF-1, which are muscle protein degradation factors that induce ubiquitination, were notably increased by TNF- $\alpha$ treatment. However, atrogin- 1 and MuRF-1 levels were restored by CME and IcA treatment compared to that of untreated cells (Fig. 2C and 2D).

Effects of CME and IcA on the loss of skeletal muscle Bodyweight, muscle mass, muscle volume, and CSA were
A

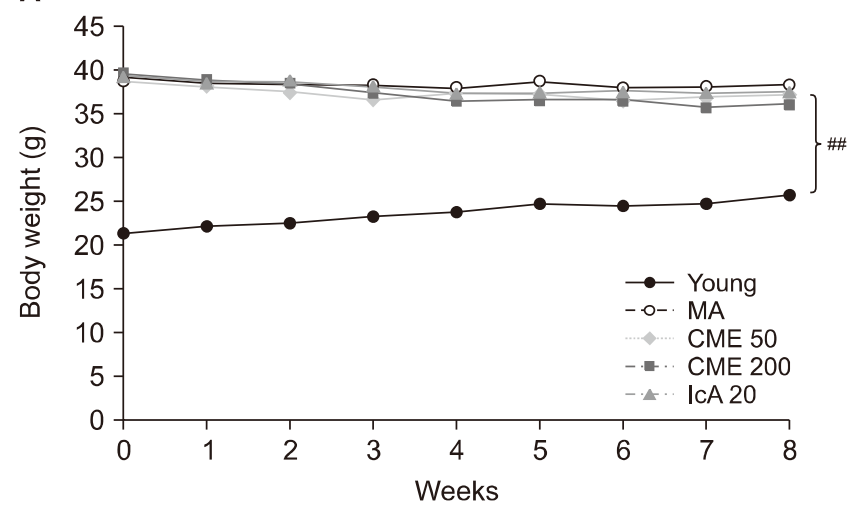

C
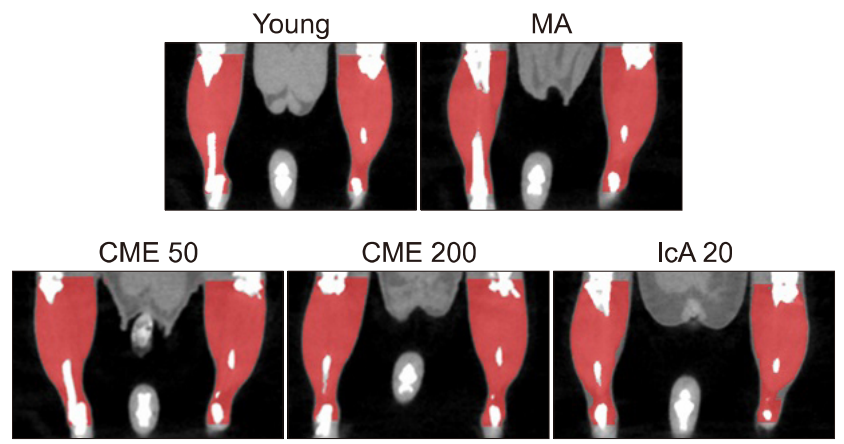

E
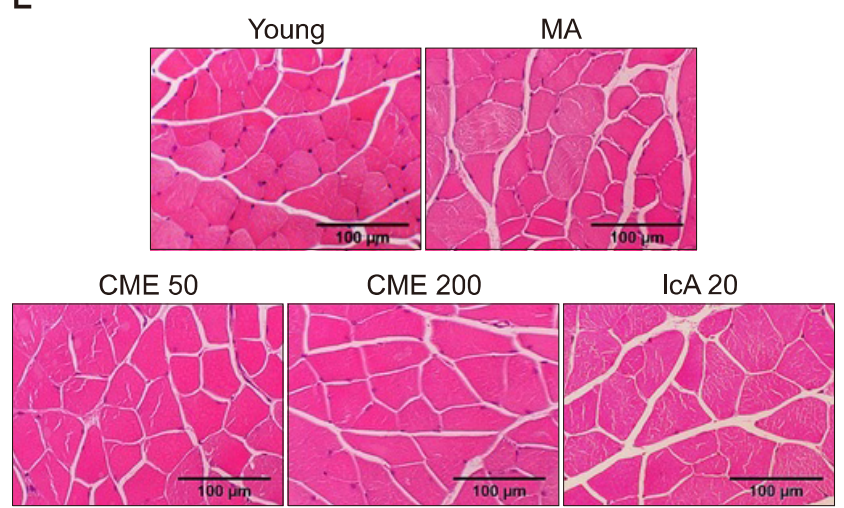

B

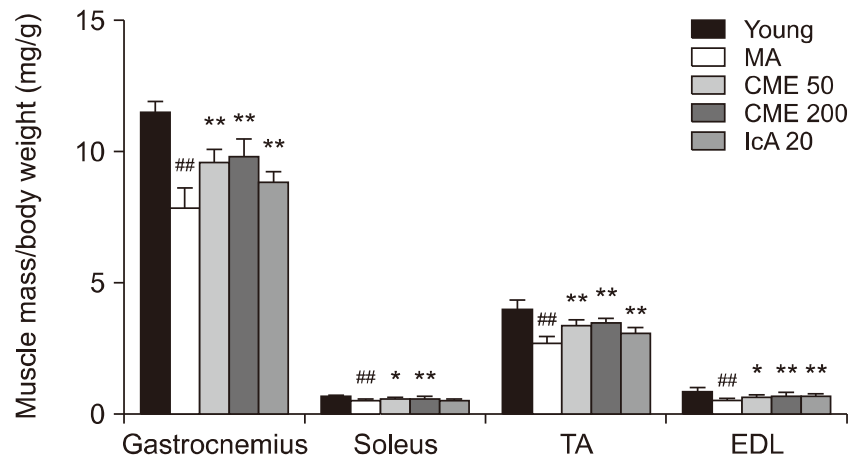

D

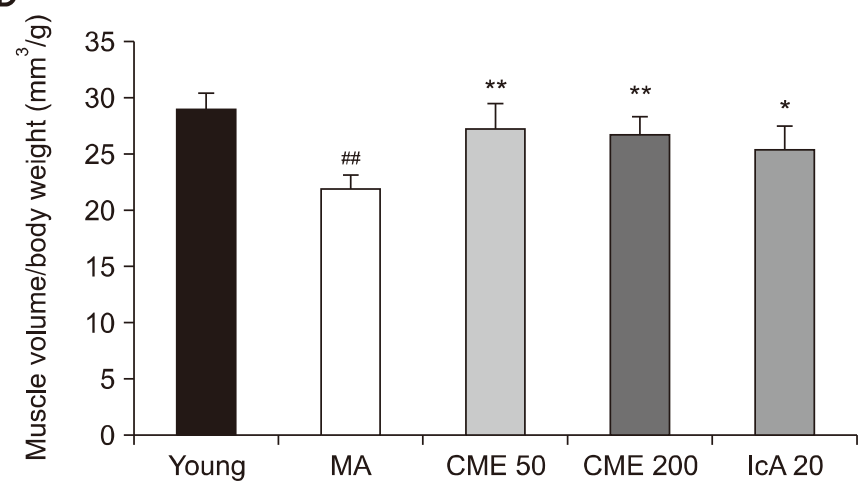

$\mathbf{F}$

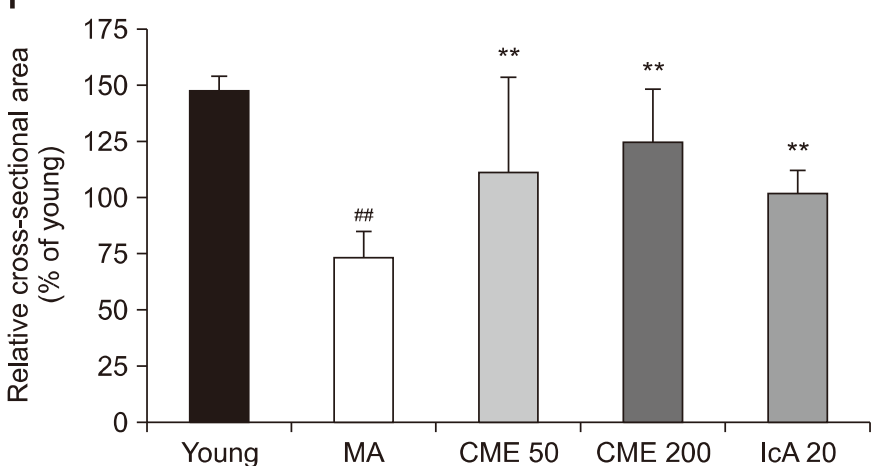

Fig. 3. Effects of Chrysanthemum morifolium Ramat. extract (CME) and isochlorogenic acid A (IcA) on body weight, skeletal muscle mass, volume, and cross-sectional area of myofibers. (A) Measured body weight and (B) masses of gastrocnemius, soleus, tibialis anterior (TA), and extensor digitorum longus (EDL), normalized to body weight. (C and D) Muscle volume of hindlimb calculated using micro-computed tomography, and ( $\mathrm{E}$ and $\mathrm{F})$ cross-sectional area of muscle fiber evaluated via H\&E staining ( $\times 200)$. Data are presented as mean \pm standard deviation. Group differences were assessed using Duncan's multiple range test. ${ }^{\# \#} P<0.01$ [young group vs. middle-aged (MA) group] and ${ }^{*} P<0.05$ and ${ }^{* *} P<0.01$ (MA group vs. CME and IcA groups). 
measured in C57BL/6J mice. Body weights were compared between groups after oral administration of CME and IcA. The MA mice had nearly 1.5 -fold increased body weight compared to the young group. However, treatment with CME and IcA did not significantly influence body weight (Fig. 3A). The normalized mass of GA, SOL, TA, and EDL muscles in the MA group were lower than those in the young group by $31.7 \%, 20.3 \%, 34.0 \%$, and $37.2 \%$, respectively. In contrast, the mass of GA, TA, and EDL muscles were significantly increased after 8 weeks of oral administration of CME and IcA (Fig. 3B). For SOL muscle, CME treatment notably increased muscle weight, while IcA treatment did not induce any significant changes. The volume of hindlimb muscle was recovered by treatment with CME and IcA, which was consistent with the muscle mass changes (Fig. 3C and 3D). The CSA of the GA muscle fibers in the MA group was reduced by $26 \%$, compared to the young group; however, this was recovered by $38 \%, 70 \%$ and $38 \%$ in the CME 50, CME 200, and IcA 20 groups, respectively (Fig. 3E and $3 \mathrm{~F})$.

\section{Effects of CME and ICA on grip strength and exercise capacity}

Muscle function was evaluated by measuring grip strength and exercise capacity. Grip strengths of the forelimb and fore/hindlimb were lower in the MA group than in the young group (Fig. 4A). Although significant differences were not observed after treatment with CME and ICA in the forelimb grip strength, fore/hindlimb grip strength was significantly increased in the CME 50, CME 200, and IcA 20 groups compared with the MA group. In particular, grip strength of the fore/hindlimb in the CME 200 group was recovered by $31.4 \%$ compared with the MA group. The exercise capacity indicated by running distance and time increased in the CME and IcA-treated groups and was consistent with the results of fore/hin- dlimb grip strength (Fig. 4B and 4C).

\section{Effects of CME and ICA on the protein turnover-related pathway}

The effects of CME and IcA on regulation of the sarcopenia-related signaling pathway were determined in vivo. In the MA group, protein levels of p-PI3K and p-Akt were considerably reduced. Oral administration of CME 200 and IcA 20 recovered p-PI3K by $164 \%$ and $92 \%$, respectively, and p-Akt protein levels by $77 \%$ and $43 \%$, respectively (Fig. 5A). Furthermore, protein levels of p-mTOR, p-p70S6K, and p-4EBP1 were upregulated by CME administration, whereas significant differences were not observed in the IcA-treated groups (Fig. 5B). Compared with the MA group, phosphorylation of FoxO3a was increased by CME and IcA (Fig. 5C). In the MA group, mRNA of the muscle degradation genes atrogin- 1 and MuRF-1 were highly expressed compared with those in the young group. However, oral administration of CME and IcA inhibited expression of atrogin-1 and MuRF-1 (Fig. 5D).

\section{DISCUSSION}

Age-related muscle atrophy, sarcopenia, induces deterioration of muscle function and mitochondria in the older population. Generation of cytokines associated with inflammation, such as TNF- $\alpha$, IL-1 $\beta$, and IL-6, also influence the occurrence of sarcopenia (Kalyani et al., 2014). In this study, we used TNF- $\alpha$-treated skeletal muscle cells and sarcopenic animal models to show that CME and IcA attenuates sarcopenia and recovers muscle function by regulating protein expression related to muscle synthesis and degradation (Fig. 1 and 5).

The amount of protein in skeletal muscle is regulated by the balance between synthesis and degradation (Sacheck
A

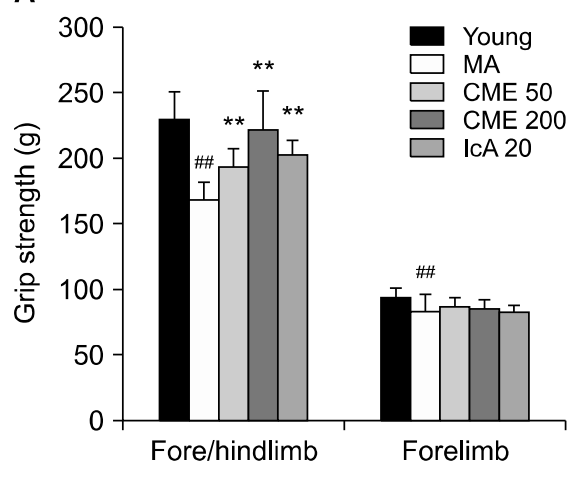

B

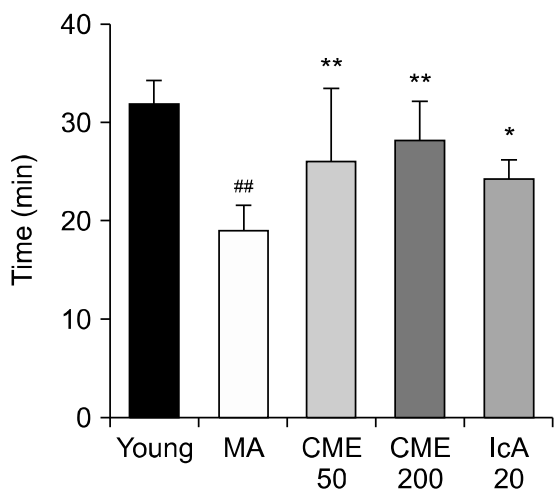

C

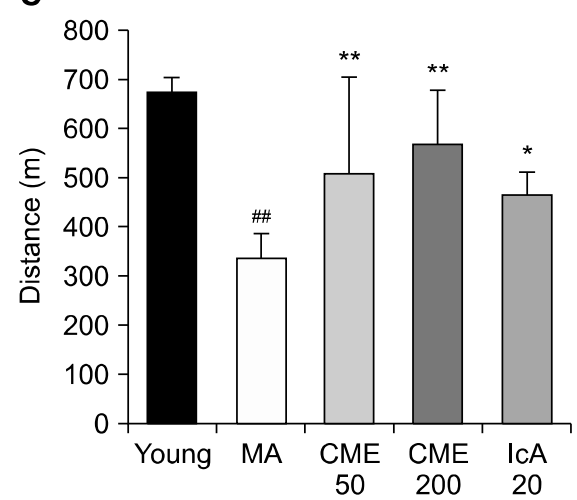

Fig. 4. Effects of Chrysanthemum morifolium Ramat. extract (CME) and isochlorogenic acid A (IcA) on grip strength and exercise capacity. (A) Grip strengths of fore/hindlimb and forelimb were measured using a grip strength meter. (B) Running time and (C) running distance were evaluated by treadmill tests. Data are presented as mean \pm standard deviation. Group differences were assessed using Duncan's multiple range test. ${ }^{\# \#} P<0.01$ [young group vs. middle-aged (MA) group] and ${ }^{*} P<0.05$ and ${ }^{* *} P<0.01$ (MA group vs. CME and IcA groups). 
A
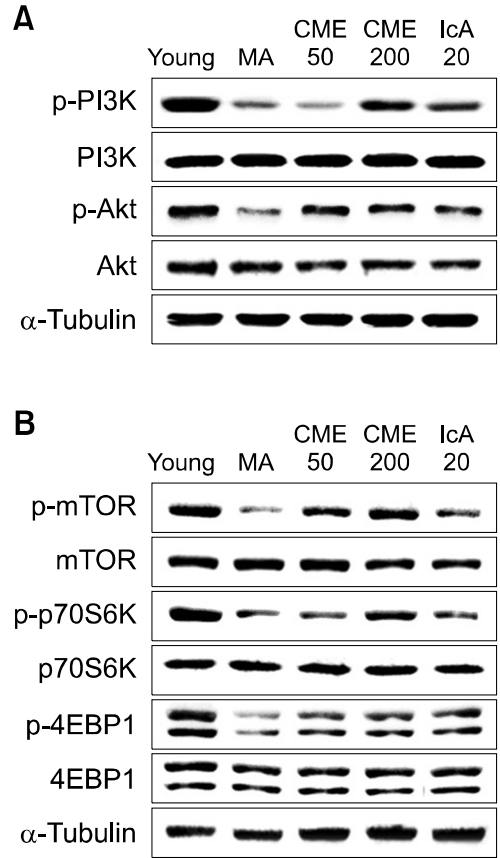

C

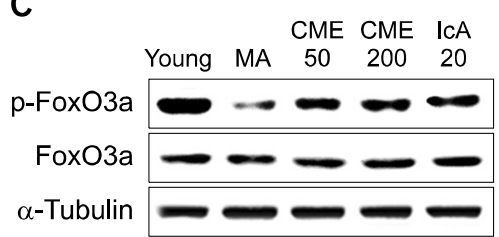

D

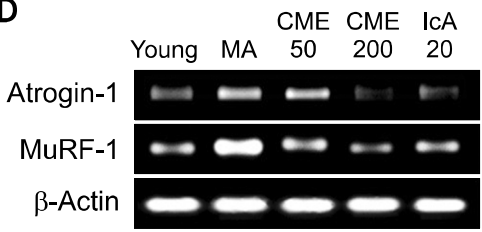

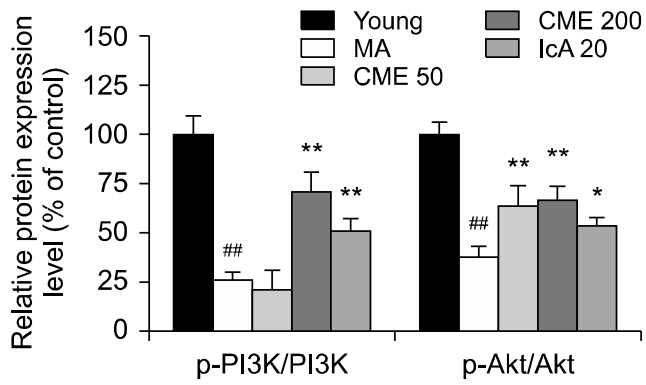
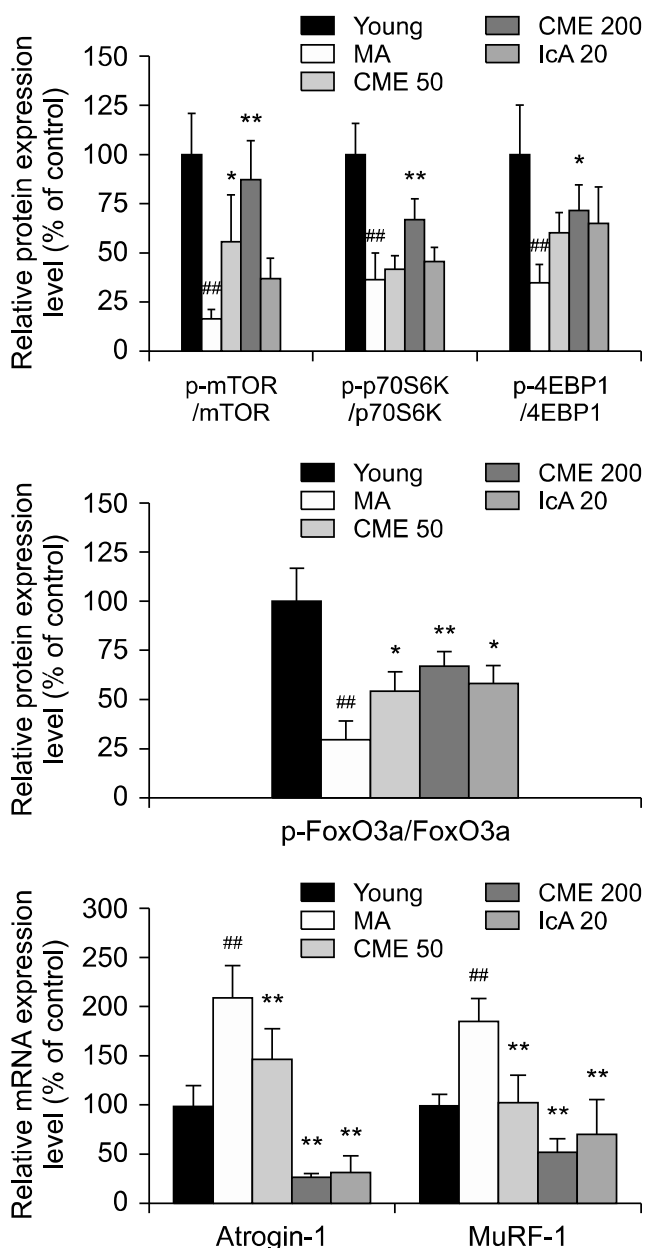

Fig. 5. Effects of Chrysanthemum morifolium Ramat. extract (CME) and isochlorogenic acid A (IcA) on protein turnover-related pathways in gastrocnemius muscle. Gastrocnemius muscle tissues were used to analyze the expression levels of protein turnover-related factors. Relative protein levels of (A) p-PI3K, PI3K, p-Akt, and Akt, (B) p-mTOR, mTOR, p-p70S6K, p70S6K, p-4EBP1, and 4EBP1, and (C) p-FoxO3a and FoxO3a measured using Western blot, with $\alpha$-tubulin as the housekeeping gene. Relative mRNA levels of (D) atrogin-1 and MuRF-1 measured using reverse transcription-polymerase chain reaction, with $\beta$-actin as the housekeeping gene. Data are presented as mean \pm standard deviation. Group differences were assessed using Duncan's multiple range test. ${ }^{\# \#} P<0.01$ [young group vs. middle-aged (MA) group] and ${ }^{*} P<0.05$ and ${ }^{* *} P<0.01$ (MA group vs. CME and IcA groups).

et al., 2004). In particular, Akt inhibits protein degradation by phosphorylating FoxO3a and promotes muscle protein synthesis by activating the mTOR pathway (Zhang et al., 2011). FoxO3a is crucial for transcribing atrogin-1 and MuRF-1, subsequently activating the ubiquitin-proteasome system (Sandri, 2013). Western blot analysis showed that CME and IcA efficiently prevent and/or recover TNF- $\alpha$-induced dephosphorylation of Akt, resulting in regulation of sub-signaling cascades, such as phospho- rylation of mTOR pathway proteins and FoxO3a. Moreover, enhanced phosphorylation of FoxO3a suppressed atrogin-1 and MuRF-1 expression, which resulted in a reduction of ubiquitin-proteasome-based protein degradation (Fig. 2 and 5). These results suggest that CME and IcA may stimulate protein synthesis and inhibit protein degradation effectively by activating Akt as a dual regulator of the mTOR pathway and FoxO3a. Although IcA rarely induced phosphorylation of Akt, mTOR, and FoxO3a 
compared with CME in vivo, it significantly reduced transcription of atrogin-1 and MuRF-1.

These molecular changes were revealed through investigating muscle mass, muscle volume, and exercise capacity. Moreover, CME and IcA inhibit progression of sarcopenia by increasing physical performance and muscle mass. Muscle fibers are classified as type I and type II muscle fibers. Type II muscle fibers are further classified as type IIA, type IIX, and type IIB based on the speed of muscle contractility, energy productivity, and expression of myosin heavy chains (Ciciliot et al., 2013). In rodents, GA, TA, and EDL muscles comprise high proportions of type II compared with type I muscle fibers. In contrast, SOL muscle has a high proportion of type I muscle fibers (Westerblad et al., 2010). In general, type I muscle fibers are involved in aerobic exercise, and type II fibers are associated with anaerobic exercise performance (Bloemberg and Quadrilatero, 2012). This study demonstrated that aerobic exercise capacity, measured by the treadmill test, was improved by an increase in SOL muscle mass in CME-treated groups (Fig. 3B, 4B, and 4C). In a previous study, the decline in skeletal muscle mass due to aging was attributed to a decrease in type II muscle fibers (Nilwik et al., 2013). Oral administration of CME and IcA increased GA muscle mass and grip strength in middleaged mice (Fig. 3B and 4A), suggesting that this could result from increased amounts of type II muscle fibers.

Sarcopenia is characterized by a reduced number of satellite cells and lowered CSA of muscle fibers. Satellite cells are necessary for regenerating muscle fibers during muscle injury or muscle atrophy. A reduction in satellite cells leads to a reduced ability to rebuild muscle tissue in skeletal muscles (Verdijk et al., 2010; Fry et al., 2015). In the present study, the CSA of muscle fibers was decreased in the MA group, but this decrease was alleviated in all the CME and IcA groups (Fig. $3 \mathrm{E}$ and $3 \mathrm{~F}$ ). $\mathrm{CME}$ and IcA may potentially improve the CSA of muscle fibers by recovering the satellite cell pool.

Collectively, these results suggest that CME and IcA could potentially be developed into functional food supplements to attenuate sarcopenia by recovering muscle mass, and enhancing exercise capacity and grip strength. At the molecular level, CME and IcA are involved in muscle hypertrophy, and the PI3K/Akt and mTOR/P70S6K/ 4EBP-1 pathways, and prevent muscle degradation via the FoxO3a pathway. To elucidate the inhibitory effects of CME and IcA on sarcopenia, further studies in humans and suitable clinical trials are necessary.

\section{ACKNOWLEDGEMENTS}

This work was supported by the World Class 300 Project R\&D Program (S2435140) funded by the Small and
Medium Business Administration (SMBA, Republic of Korea).

\section{AUTHOR DISCLOSURE STATEMENT}

The authors declare no conflict of interest.

\section{REFERENCES}

Abushouk AI, Negida A, Ahmed H, Abdel-Daim MM. Neuroprotective mechanisms of plant extracts against MPTP induced neurotoxicity: future applications in Parkinson's disease. Biomed Pharmacother. 2017. 85:635-645.

Bloemberg D, Quadrilatero J. Rapid determination of myosin heavy chain expression in rat, mouse, and human skeletal muscle using multicolor immunofluorescence analysis. PLoS One. 2012. 7:e35273. https://doi.org/10.1371/journal.pone. 0035273

Chen L, Kotani A, Kusu F, Wang Z, Zhu J, Hakamata H. Quantitative comparison of caffeoylquinic acids and flavonoids in Chrysanthemum morifolium flowers and their sulfur-fumigated products by three-channel liquid chromatography with electrochemical detection. Chem Pharm Bull (Tokyo). 2015. 63:2532.

Ciciliot S, Rossi AC, Dyar KA, Blaauw B, Schiaffino S. Muscle type and fiber type specificity in muscle wasting. Int J Biochem Cell Biol. 2013. 45:2191-2199.

Cui Y, Wang X, Xue J, Liu J, Xie M. Chrysanthemum morifolium extract attenuates high-fat milk-induced fatty liver through peroxisome proliferator-activated receptor $\alpha$-mediated mechanism in mice. Nutr Res. 2014. 34:268-275.

Frontera WR, Ochala J. Skeletal muscle: a brief review of structure and function. Calcif Tissue Int. 2015. 96:183-195.

Fry CS, Lee JD, Mula J, Kirby TJ, Jackson JR, Liu F, et al. Inducible depletion of satellite cells in adult, sedentary mice impairs muscle regenerative capacity without affecting sarcopenia. Nat Med. 2015. 21:76-80.

Ge Y, Chen J. Mammalian target of rapamycin (mTOR) signaling network in skeletal myogenesis. J Biol Chem. 2012. 287:4392843935.

Hong S, Joo T, Jhoo JW. Antioxidant and anti-inflammatory activities of 3,5-dicaffeoylquinic acid isolated from Ligularia fischeri leaves. Food Sci Biotechnol. 2015. 24:257-263.

Joseph AM, Adhihetty PJ, Buford TW, Wohlgemuth SE, Lees HA, Nguyen LM, et al. The impact of aging on mitochondrial function and biogenesis pathways in skeletal muscle of sedentary high- and low-functioning elderly individuals. Aging Cell. 2012. 11:801-809.

Kalyani RR, Corriere M, Ferrucci L. Age-related and disease-related muscle loss: the effect of diabetes, obesity, and other diseases. Lancet Diabetes Endocrinol. 2014. 2:819-829.

Kim SJ. Herbal chrysanthemi flos, oxidative damage and protection against diabetic complications. In: Preedy VR, editor. Diabetes: Oxidative Stress and Dietary Antioxidants. Academic Press, London, UK. 2014. p 201-211.

Kim SS, Park RY, Jeon HJ, Kwon YS, Chun W. Neuroprotective effects of 3,5-dicaffeoylquinic acid on hydrogen peroxide-induced cell death in SH-SY5Y cells. Phytother Res. 2005. 19: 243-245.

Lagirand-Cantaloube J, Cornille K, Csibi A, Batonnet-Pichon S, Leibovitch MP, Leibovitch SA. Inhibition of atrogin-1/MAFbx mediated MyoD proteolysis prevents skeletal muscle atrophy 
in vivo. PLoS One. 2009. 4:e4973. https://doi.org/10.1371/ journal.pone.0004973

Misra A, Singhal N, Khurana L. Obesity, the metabolic syndrome, and type 2 diabetes in developing countries: role of dietary fats and oils. J Am Coll Nutr. 2010. 29:289S-301S.

Nilwik R, Snijders T, Leenders M, Groen BB, van Kranenburg J, Verdijk LB, et al. The decline in skeletal muscle mass with aging is mainly attributed to a reduction in type II muscle fiber size. Exp Gerontol. 2013. 48:492-498.

Sacheck JM, Ohtsuka A, McLary SC, Goldberg AL. IGF-I stimulates muscle growth by suppressing protein breakdown and expression of atrophy-related ubiquitin ligases, atrogin- 1 and MuRF1. Am J Physiol Endocrinol Metab. 2004. 287:E591-E601.

Sanchez AM, Candau RB, Bernardi H. FoxO transcription factors: their roles in the maintenance of skeletal muscle homeostasis. Cell Mol Life Sci. 2014. 71:1657-1671.

Sandri M. Protein breakdown in muscle wasting: role of autophagy-lysosome and ubiquitin-proteasome. Int J Biochem Cell Biol. 2013. 45:2121-2129.
Schiaffino S, Dyar KA, Ciciliot S, Blaauw B, Sandri M. Mechanisms regulating skeletal muscle growth and atrophy. FEBS J. 2013. 280:4294-4314.

Verdijk LB, Snijders T, Beelen M, Savelberg HH, Meijer K, Kuipers $\mathrm{H}$, et al. Characteristics of muscle fiber type are predictive of skeletal muscle mass and strength in elderly men. J Am Geriatr Soc. 2010. 58:2069-2075.

Westerblad H, Bruton JD, Katz A. Skeletal muscle: energy metabolism, fiber types, fatigue and adaptability. Exp Cell Res. 2010. 316:3093-3099.

Wolfe RR. The underappreciated role of muscle in health and disease. Am J Clin Nutr. 2006. 84:475-482.

Yamamoto J, Tadaishi M, Yamane T, Oishi Y, Shimizu M, Kobayashi-Hattori K. Hot water extracts of edible Chrysanthemum morifolium Ramat. exert antidiabetic effects in obese diabetic KK-Ay mice. Biosci Biotechnol Biochem. 2015. 79:1147-1154. Zhang X, Tang N, Hadden TJ, Rishi AK. Akt, FoxO and regulation of apoptosis. Biochim Biophys Acta. 2011. 1813:1978-1986. 